\title{
Relative distributions of cosmic ray electrons and magnetic fields in the ISM
}

\author{
Rodion Stepanov ${ }^{1}$, A. Fletcher ${ }^{2}$, A. Shukurov ${ }^{2}$, R. Beck ${ }^{3}$, \\ L. La Porta ${ }^{3}$, and F. S. Tabatabaei ${ }^{3}$ \\ ${ }^{1}$ Institute of Continuous Media Mechanics, 1 Korolyov St., Perm 614013, Russia \\ ${ }^{2}$ School of Mathematics and Statistics, Newcastle University, NE1 7RU, UK \\ ${ }^{3}$ Max-Planck-Institut für Radioastronomie, Auf dem Hügel 69, 53121 Bonn, Germany
}

\begin{abstract}
We calculate the relative magnitudes of the fluctuations in total synchrotron intensity in the interstellar medium, both from observations and from theory under various assumptions about the correlation or anticorrelation between cosmic rays and interstellar magnetic fields. The results are inconsistent with local energy equipartition between cosmic rays and magnetic fields. The distribution of cosmic rays must be rather uniform at scales of order $1 \mathrm{kpc}$, whereas interstellar magnetic fields vary at much smaller scales.
\end{abstract}

Keywords. Cosmic rays - magnetic fields - radio continuum

The concept of energy equipartition between cosmic rays and magnetic fields is often used in the analysis of radio astronomical observations (Beck \& Krause 2005). There is no sound basis for this assumption, but as cosmic rays are confined by magnetic fields, it seems natural to expect some relation between them, though this does not emerge from cosmic ray propagation models (e.g., Padoan \& Scalo 2005). Here we test the equipartition hypothesis using models of the non-thermal interstellar medium (ISM) and radio observations. We show that equipartition is inconsistent with observations at scales of order $0.1 \mathrm{kpc}$ (assuming that cosmic ray protons and electron are similarly distributed).

We used radio maps of the Milky Way and the nearby galaxy M 33 to calculate the standard deviation and mean value of the total synchrotron intensity in a range of scales. The data used are: (i) the $408 \mathrm{MHz}$ all-sky survey of Haslam et al. (1982) at the resolution of $50^{\prime}$ (upper left panel in Fig. 1), (ii) the $22 \mathrm{MHz}$ survey of Roger et al. (1999) at a resolution of approximately $1^{\circ} \times 2^{\circ}$, and (iii) the synchrotron $1.4 \mathrm{GHz}$ map of M 33 (Tabatabaei et al. 2007) at a resolution of $90^{\prime \prime}$ shown in Fig. 1 (right-hand panel).

At a distance of $1 \mathrm{kpc}$, the angular diameter of a turbulent cell of $0.1 \mathrm{kpc}$ in size is $6^{\circ}$. Thus, the nearest turbulent cells are resolved in both Milky Way maps used. At both $408 \mathrm{MHz}$ and $22 \mathrm{MHz}$, the emission is predominantly synchrotron radiation. At the distance of $\mathrm{M} 33$, the resolution of our data is $0.4 \mathrm{kpc}$. We considered eight rectangular regions which are free of bright sources (Fig. 1). Since the regions are several kpc across, we removed large-scale linear and quadratic trends in synchrotron intensity in each region.

The standard deviation $\sigma_{I}$ and the mean value $\bar{I}$ of the synchrotron intensity were calculated for each map using sliding averaging with a Gaussian kernel of angular radius $a$. For random fluctuations, the ratio $\sigma_{I} / \bar{I}$ is independent of $a$ for $a \gg a_{0}$, where $a_{0}$, the angular radius of a turbulent cell, corresponds to $0.05 \mathrm{kpc}$. This is indeed the case and, remarkably, the relative fluctuations of the synchrotron intensity have rather similar magnitudes in all the data sets considered, $\sigma_{I} / \bar{I} \lesssim 0.2$. We believe that this value is not significantly affected by either regular trends in the radio intensity, nor by discrete radio 

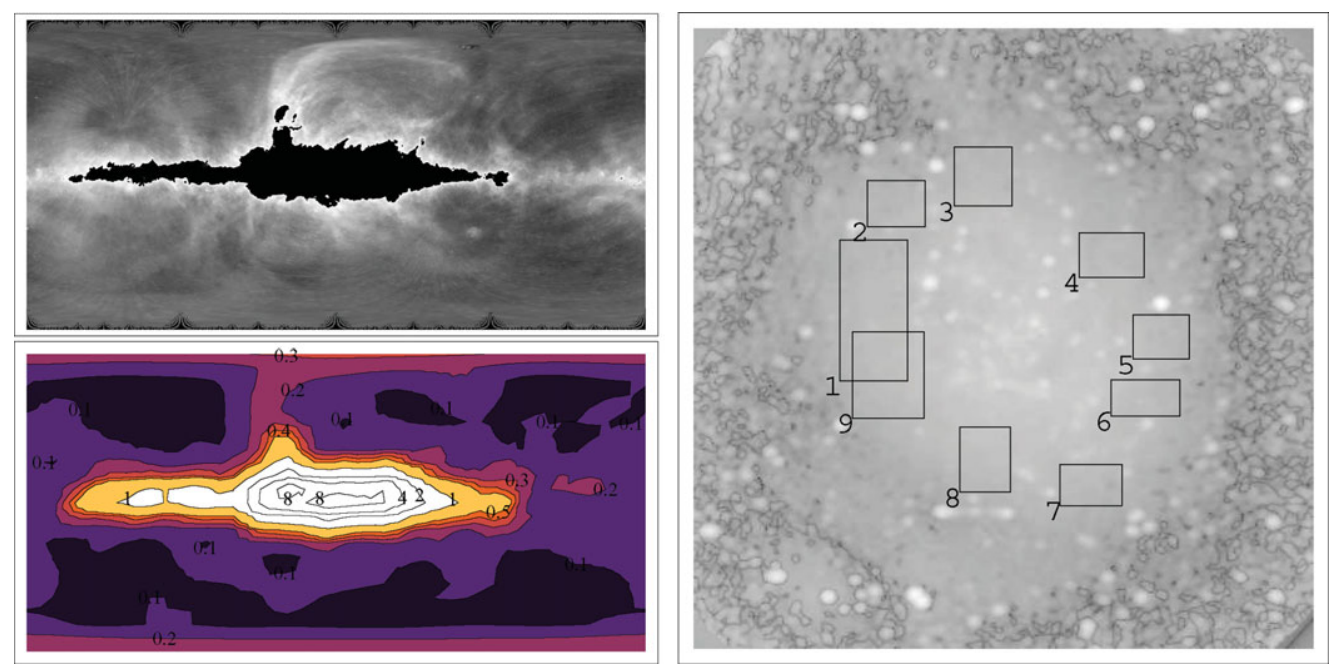

Figure 1. Upper left: the $408 \mathrm{MHz}$ all-sky map with the strongest discrete sources removed by La Porta et al. (2008). Lower left: $\sigma_{I} / \bar{I}$ from the data smoothed at the scale (radius) $a=7^{\circ}$. Right panel: the synchrotron $1.4 \mathrm{GHz}$ map of M 33 with the regions used in our analysis. Note the smoothness of the images out of the Milky Way plane and across M 33: here $\sigma_{I} / \bar{I} \lesssim 0.2$.

sources or thermal emission. Anyway, the above estimate is a firm upper limit of the level of fluctuations in synchrotron intensity arising in the ISM of the Milky Way and M33.

We model the non-thermal ISM by specifying a divergence-free magnetic field $\mathbf{B}$, with a given power spectrum of isotropic fluctuations and a mean value $B_{0}$, in terms of a Fourier series with randomly chosen parameters. Cosmic ray electron density $n$ (also represented by a mean value and fluctuations) is then similarly introduced, with a given cross-correlation coefficient $C$ between $n$ and $B^{2}$, with $|C| \leqslant 1$. Synthetic radio maps are then calculated with a range of $C$ and a range of relative fluctuation amplitudes in $\mathbf{B}$ and $n$. The overall conclusion is that $\sigma_{I} / \bar{I}$ is as observed only if $-1 \lesssim C<0$.

Thus, observations appear to rule out any positive correlation between magnetic fields and cosmic rays at scales of order $0.1 \mathrm{kpc}$. Interpretations that rely on this or similar assumptions seriously underestimate magnetic field fluctuations and overestimate those in cosmic rays. However, some form of equipartition may still be maintained at scales of $1 \mathrm{kpc}$ or larger. We suggest an alternative approach to the interpretation of radio maps, where they are first smoothed to a linear resolution of about $1 \mathrm{kpc}$, and the large-scale distributions of magnetic fields and cosmic rays may be obtainable from the equipartition argument. The cosmic ray distribution thus obtained can be used together with the original synchrotron data to recover a full-resolution picture of magnetic fields.

This work was supported by the STFC grant F003080/1, the NSF Grant NSF PHY0551164 and by grant RFBR-08-02-92881.

\section{References}

Beck, R. \& Krause, M. 2005, AN 326, 414

Haslam, C. G. T., Salter, C. J., Stoffel, H., \& Wilson, W. E. 1982, A\&AS 47, 1

La Porta, L., Burigana, C., Reich, W., \& Reich, P. 2008, A\&SA 479, 641

Padoan, P. \& Scalo, J. 2005, ApJ 624, L97

Roger, R. S., Costain, C. H., Landecker, T. L., \& Swerdlyk, C. M. 1999, A\&AS 137, 7

Tabatabaei, F. S., Beck, R., Krause, M., et al. 2007, A\&\&A 475, 133 